more right to assign etiological significance to uric acid than to. other substances of an unknown nature found in the structureless material left after removal of the crystals.

(b) An examination of gonty tendons showed that the necrotic areas had a definite relation to the vessels of the part. The appearances in the tendons indicate that the necrotic areas are to be accounted for by an infection by the blood stream.

(c) The erosion of cartilage was due not to the presence of uric acid but to the action of small rounds cells of the granulation tissue type.

(d) At the gouty deposits in the bone, attention was directed to (1) the richness of the blood supply, (2) the general character of the giant cells, and (3) the great accumulation of emall round cells of the nature commonly associated with the action of bacterial toxins.

(e) Stress was laid on the alterations in the interior of the bone, more especially on the disappearance of the marrow proper and rarefaction of the bone trabeculae.

The general conclusion in this paper was that "the tout ensemble of the pathological pictures is strikingly similar to that seen in chronic infective diseases. From this point of view the uric acid is regarded as the feature which gives the inflammation its specific character." A study of the facts recorded in this and the preceding paper showed to my mind conclusively that the adoption of the uric acid theory had obscured the significance of the facts actually observed.

Observation VI.-Frcm the Clinical Standpoint.

When it became apparent that the results obtained from these histological investigations did not support the uric-acid theory, but rather suggested an infective condition, the point at once presented itself for consideration whether the latter hypothesis would conflict with the teaching of clinical experience. It does not. I submit that, on the contrary, this hypothesis more adequately explains the phenomena of acute gout than does any other theory.

Dr. Woods. Hutchinson rightly lays stress on leucocytosis. the main bone-marrow function. The importance of this function and the correctness of Dr. Woods Hutchinson's conception of gout have been strongly emphasized to me by the results of some clinical and therapeutic observations on bone marrow which I have carried out coincidently with the investigations referred to in this paper.

It only remains for me to refer to the likely lines for fruitful investigation. Reliable evidence for or against the general theory put forward in my paper should, I think, be looked for along the following lines:

(a) It is advisable to investigate the influence of ( 1 ) a meat diet and (2) a carbohydrate diet, on the digestive secretions and on the ductless glands. Special attention should be directed to the thyroid gland and the bone marrow.

(b) It appears advisable to repeat Ebstein's experiments with the aid of skilled bacteriological methods.

\title{
REFERENCES
}

1 BRitish Medical Journal, January 28th, I899. 2 Ibid., January 6th, rgoo. 3 Ibid ; also Journal of Pathology and Bacteriology, December, rgo0. 4 BRITISH MEdical JoURNal, January 9th, Ig04. 5 Ibid., July r6th, 1904

\section{A NOTE ON SENILE SYMMETRICAL ATROPHY OF THE SKULL.}

Bx F. PARKES WEBER, M.D., F.R.C.P., Physician to the German Hospital.

A curIous condition of symmetrical circumscribed thinning of the parietal bones, generally regarded as a form of senile atrophy of the skull, has been described, with or without illustrations, by Virchow, ${ }^{1}$ Maier, ${ }^{2}$ Sauvage, ${ }^{3}$ Mejer, ${ }^{4}$ Féré, Eve $^{6}$ Humphry, ${ }^{7}$ and others. Most examples have occurred in old persons, especially women, but Meyer mentions commencing atrophy of the parietal bones in a melancholic man only 44 zears of age, and I have met with a typical instance in a woman only 55 years old, the subject of biliary cirrhosis and jaundice of four years' duration, whose case I described in the BRITIsH Medical Jodrnal for April 25th, 1896. In all cases the change in the skull is the same in kind, the only difference consisting in the degree of thinning of the parietal bones and in the size of the thinned areas. The thinning is due to a circumscribed depression with shelving edge on the outer surface of the parietal bone, due, as Mr. Targett ${ }^{8}$ pointed out in the specimen I gave to the Royal College of Surgeons, to

In the clinical picture of acute gout, the manner of onset, the course of the temperature curve, the changes in the blood indicating disturbances of the marrow function, ${ }^{2}$ and the marked liability to relapses, all strikingly recall the picture of an acute infective disorder. This is illustrated in the chart of one of the cases recorded in Observation III.

Generat Conclusions.

From the foregoing we conclude that the evidence of the laboratory is in harmony with the teaching of clinical experience, and shows that uric acid is not an important etiological factor in the production of gout. The author believes that (I) there is an infective element in the disease; and (2) the uric acid is the feature which gives the inflammation its specific character. According to this view the chief source of infection is the alimentary tract, and an injudicious dietary-meat or drink-acts mainly in virtue of its influence on the bacteria present in the digestive tract. This view by no means minimizes the importance of the hereditary factor in the disease.

In concluding this summary, I must refer to a very suggestive paper on gout recently published in the Lancet by Woods Hutchinson. The keynote of this paper is, I think, to be found in its definition of gout as a "Toxaemia of gastro-intestinal origin accompanied by the formation of an excess of urates, this excess of urates being due to the breaking down of the leucocytes and fixed cells in the attempt to neutralize the poison-in other words, being the measure of the resisting power of the tissues. The formation and introduction of the toxins, be it well understood, are by no means confined to the gouty; it is only the nature of the resistance of the body to them that gives the character of gout." removal of the onter table over the affected area and
wearing away of the diploë, so that the inner table forms the floor of the depression. The inner surface of the skull is generally unaltered.

In the museum of the Royal College of Surgeons are Egyptian and other skulls showing these symmetrical areas of atrophy. One of these skulıs, presented to the museum by Mr. Flinders Petrie, is from Gurob (Fayoum) in Egypt, probably of about 1,300 years or so belore the Christian era, and has been described and figured by Mr. F. S. Eve in the Transactions of the Pathological Society of London for 1890 . Sir G. M Humphry, ${ }^{9}$ who, in his book on the Human Skeleton, in 1858 attributed the condition to a peculiarity of congenital conformation, afterwards, in 1890 , before the $R$ ijal Medical and Chirurgical Society of London, described it as probably a result of senile changes. Ziegler, ${ }^{10}$ in the ninth German edition of his well-known textbook of pathological anatomy, gives an excellent illustration of symmetrical parietal depressions, and thus refers to the subject: "Lacunar absorption affecting large portions or the whole of the skeleton takes place first in old age, and is, therefore, called senile atrophy. The process is sometimes especially advanced in the flat bones of the body, the skull, the shoulder-blades, and the pelvis, notably in parts which are not covered by muscles. In the skull the absorption of the parietal bones may proceed so far that the whole of the outer table and the diploë become destroyed, and finally portions of the inner table, and at spots here and there even both tables, completely disappear."

But the question arises, Is this symmetrical atrophy of parietal bones merely a senile change, and if so, why does it sometimes occur in younger persons? Bernard Hollander ${ }^{11}$ collected a number of cases associated with melancholia, and regarded the condition as due to trophic influences accom- 
panying the melancholic state. I think there can be no doubt that the change is due more to senility than anything else, and would express myself thus :

The condition is probably due to a process of lacunar absorption accompanying senility or other chronic states of depressed nutrition.

Not every senile change is present in every aged person, and some senile changes develop relatively early in life in individuals predisposed to them by hereditary factors, or by wasting diseases or chronic causes of depressed nutrition, including mental causes-for example, premature whitening of the hair.

These considerations may serve to account for the early onset of symmetrical atrophy of the parietal bones in a patient with melancholia, as also in my case of chronic jaundice and cirrhosis of the liver. In the same way " brown atrophy" of the heart, exactly similar to that found in very aged persons, may notoriously sometimes be met with in younger persons dying of cancer and other chronic wasting disorders.

The lacunar absorption which causes the atrophy of the outer table in the parietal bones is probably not solely due to senility, but, as other " genile" atrophies, intimately connected with it. Senile atrophies of organs and parts of the body are nearly always either general or symmetrical in distribution. The localization of symmetrical senile atrophies is probably to be accounted for by portions being selected which are no longer being much used, and are of least vital importance for the organism. Thus the bones and muscles of extremities which are not much used tend to atrophy early, and in the skull the symmetrical atrophy of the parietal bones occupies a site which is not pulled on by muscles, and is mechanically of relatively little importance for maintaining the strength of the brain case and its safety from concussion.

VIr. REFERENCES.

1 Virchow, Verhandl. d. phys.-med. Gesellsch. in Wïrzburg, 1854, vol. iv p. 354. 'Maier, Virchow's Arch., 1854, vol. vii, p. 336. 'sauvage, Recherche sur l'Etat Sénile du Cráne, Paris, 1870. $4 \mathrm{~L}$. Meyer, Arch. für Psychiatrie, Berlin, 1872, vol. ili, p. ${ }^{15}$. ${ }^{5}$ Ch. Fere, Bull. de la soc. Anat. Paris, 188 vol. vi, pp. 414, 472. 2 . S. Eve, Trans. Path. Soc. London, 1890, vol. xli, p. 242 Humphry, The Human Skeleton, Cambridge, 1858, p. 242, and Med.Trans., 1890, vol. 1xxili, p. 327. Targett, Description of the Calvarium

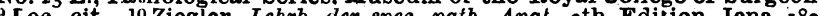
p. r17. 11 Bernard Hollander, Journ. of Mental Science, 1901, vol. xlvii, p. 475

\section{ON THE SIGNIFICANCE OF SCARS OF THE GENITAL REGION: IN THE RETROSPECTIVE DIAGNOSIS OF SYPHILIS.}

\section{BY ARTHUR COOPER,}

Consulting Surgeon to the Westminster General Dispensary.

IN seeking evidence of syphilis in cases of nervous or other internal disease when the cause is obscure, it is usual to examine the skin and mucous membrane, and, if scars are found in the genital region, their presence seems often to be looked on as evidence in favour of syphilis. If, on the other hand, no scars are found, their absence is sometimes looked on as implying absence of syphilis. In either case such a conclusion without further inquiry may lead to error.

In order to understand the matter aright, it is well in the first place to bear in mind what seems often to be forgotten, namely, that the primary lesion of syphilis is not in itself a destructive lesion, and therefore does not necessarily leave any scar at all. It may or may not do so according to circumstances which will be considered by and by.

Although venereal disease in some form is the chief source of genital scars it is by no means the only one, for scars in this region may be due to many other causes-such as balanitis, irregular herpes, abscesses, and injuries of various kinds, including the use of caustics and surgical operations.

Supposing, then, that a scar or scars are present on the penis or groins, or both. what is their significance, and especially what is their value as evidence of syphilis? To find an answer to this question the first thing to be done is, of course, to inquire into the history, and this in many cases will settle the point. But in many other, and notably in some venereal cases, the history helps little or not at all, so that an attempt has to be made to gain information by other means.

It has already been mentioned that the primary lesion of syphilis does not necessarily leave a scar, and when the site of inoculation is mucous membrane, as it is in the great majority of cases, and especially if the sore be under cover of the prepuce, and not cauterized or otherwise irritated, no permanent mark may be left behind. That this really happens any one who doubts may satisfy himself by examining his syphilitic patients from time to time after the primary sore has healed, when it will be found that in some of them no trace of it remains. The same remark applies also in many cases of late internal syphilitic disease. This absence of scar occurs mostly among those who are of careful and cleanly habits, and thus is oftenest met with in private practice. In many other cases, however, and in hospital practice as a general rule, pyogenic matter of some kind gains access to the primary sore, and thus a degree of ulceration, which varies in different cases, is set up, and consequently a scar may be left. But although the primary lesion of syphilis on mucous membrane may not leave a scar, it is, nevertheless, a fact that when the skin is its seat there is nearly always sufficient loss of tissue to lead to scarring, so it becomes important to remember that a scar on the body of the penis, if from a venereal sore at all, nearly always means syphilis.

The local or soft chancre is probably the principal cause of genital scars, and owing to the irritating quality and the ready autoinoculability of chancrous pus the ulcers usually multiply, and thus multiple scars remain. Exceptions to this are met with when the virus is destroyed while the sore remains single or when a careful patient manages to prevent autoinoculation. The local chancre-in the uncircumcised, at any rate-nearly always attacks primarily the mucous membrane. When the skin is affected it is usually by consecutive inoculation. When the virus of syphilis and that of the local chancre are both present the resulting. " mixed chancres" may, of course, be multiple; and, of course, also, if more than one place happen to be inoculated at the time syphilis is contracted, there may be more than one primary sore. In practice, however, it is found that the primary lesion of syphilis is single in the great majority of cases. For instance, of 103 syphilitic patients in whom the primary lesion was still present at the time they were admitted into the Male Lock Hospital when I was housesurgeon there I noted that 91 had only one sore. Of the remaining 12, 7 had two sores, and only 5 had more than two. As a general rule, therefore, it may be said that a single scar of the penis points towards syphilis, while multiple scars point to the local chancre.

A syphilitic scar, after the induration has disappeared, is generally round or oval, thin, non-adherent, and quite supple. On the skin it is also often deeply pigmented at first and the pigmentation is slow to disappear.

Gummata may, of course, leave scars either on the penis or groins, but, excepting what is sometimes called "relapsing chancre," their rarity and the time of their appearance, being themselves late signs, would make them little likely to cause difficulty in such cases as those now under consideration.

In connexion with this subject it may, be as well to mention that, apart from gumma, enlarged inguinal glands go for nothing or little in the diagnosis of late syphilis. It may also be remarked that neither in primary nor in secondary syphilis is the adenopathy of very long duration, and even at the time it is present requires to be considered in relation to other evidence, for, as has been pointed out elsewhere, ${ }^{1}$ a large number of non-syphilitic men have a degree of glandular enlargement equal to that found in some cases of primary syphilis.

If phagedaena attacks either syphilitic or local sores the resulting scar will be irregular in form and much larger than that due to almost any other cause except extensive injury, and there may be so much loss of substance that considerable deformity is left. So that very extensive scarring of penis or groins, if not traumatic, points to phagedaena.

Gonorrhoea may be accountable for scars of the penis or groins as the result of abscesses in connexion with that disease.

The scarring left by ulcerating balanitis may be considerable, and is usually accompanied by adhesions between the prepuce and glans penis. In adult life balano-posthitis is mostly arrested before it ulcerates sufficiently to leave scars, but during infancy and childhood it is often unnoticed and therefore untreated, and its effects often lead to trouble later in life, for the irregular cicatricial surface left by partial adhesions is easily abraded or torn during coitus, and is thus very liable to become inoculated if the patient is exposed to infection. Unless they become infected, however, such scars are hardly likely to lead to difficulty in diagnosis, as their appearance and history would usually prevent mistakes. 